\title{
LOGÍSTICA REVERSA E TEORIA INSTITUCIONAL: UM ESTUDO BIBLIOMÉTRICO
}

\author{
REVERSE LOGISTICS AND INSTITUTIONAL THEORY: A BIBLIOMETRIC STUDY
}

\author{
Recebido em 11.12.2019 Aprovado em 16.12.2019 \\ Avaliado pelo sistema double blind review \\ DOI: https://doi.org/10.32888/cge.v7i3.39887
}

Janinne da Silva Bitencourt

\begin{abstract}
janinnebitencourt@id.uff.br
\end{abstract}
Programa de Pós-Graduação em Administração/Universidade Federal Fluminense - Volta Redonda/RJ, Brasil

\section{Cecilia Toledo Hernández}

ctoledo@id.uff.br

Programa de Pós-Graduação em Administração/Universidade Federal Fluminense - Volta Redonda/RJ, Brasil

\section{Resumo}

Vários assuntos pertencentes a temática sustentável se destaca quanto a sua implementação no ambiente organizacional, devido a uma maior conscientização de todos os agentes. Neste cenário, destaca-se a logística reversa, área da logística que se preocupa com o ciclo de vida dos produtos, como estes são utilizados, descartados e retornados. Entretanto, a abordagem da logística reversa com a teoria institucional ainda está em estágio inicial, principalmente no cenário nacional. Para ajudar a suprir a falta de estudos acadêmicos no Brasil, este artigo possui como objetivo, fazer o mapeamento de todos os aspectos e facetas da Logística Reversa sob o olhar da Teoria Institucional, usando uma revisão estruturada da literatura para identificar orientações importantes para pesquisas futuras nesta temática. Para isto, foi realizado uma pesquisa na Web of Science e Scopus de artigos em inglês, sem especificação de tempo, com as palavras chaves Institutional Theory and Reverse Logistics. Com a pesquisa, foram encontrados 24 artigos em sua maioria de origem americana, não sendo encontrado com estes critérios nenhum artigo brasileiro. Destes foram destacadas suas informações gerais, seus periódicos de destaque e as principais características dos autores. Como recomendações futuras, pesquisas bibliométricas podem ser feitas utilizando-se de mais bases de dados.

Palavras-chave: Logística Reversa. Teoria Institucional. Sustentabilidade.

\section{Abstract}

Several issues related to sustainable themes stand out regarding their implementation in the organizational environment, due to a greater awareness of all agents. In this scenario, there is reverse logistics, an area of logistics that is concerned with the life cycle of products, how they are used, discarded and returned. However, the reverse logistics approach with the institutional theory is still at an early stage, especially in the national scenario. To help overcome the lack of academic studies in Brazil, this article aims to map all aspects and facets of reverse logistics from the perspective of institutional theory, using a structured review of the literature to identify important guidelines for future research on this topic. For this purpose, a research was conducted on Web of Science and Scopus sites, in English and without time specification, with the keywords Institutional Theory and Reverse Logistics. With the search, 24 articles were found, most of them of American origin, and no Brazilian article was found with these criteria. General information, journals and the main characteristics of the authors were extracted from them. As future recommendations, bibliometric research can be done using more databases.

Keywords: Reverse Logistics. Institutional Theory. Sustainability. 


\section{Introdução}

O acelerado crescimento industrial e econômico nos últimos anos exige continuamente que as empresas sejam capazes de oferecer produtos e serviços inovadores aos seus consumidores, com grande variedade de produtos e assim, menores ciclos de vida. Além disto, estas devem priorizar as necessidades dos clientes, não pensando somente no uso dos produtos, mas em como estes são descartados (SUBRAMONIAM; HUISINGH; CHINNAM, 2010). Como resultado deste acelerado crescimento, o meio ambiente vem sofrendo uma grande carga, devido à imensa interação entre pessoas, bens e informações, não conseguindo se auto recuperar em muitos dos casos (YE et al., 2013). Neste cenário, percebe-se que a economia mundial passa por um processo de globalização que é intensificada por diversos agentes, o que aumenta a conscientização relacionada a questões que envolve sustentabilidade, fazendo com que a sociedade, governo, consumidores e empresas adotem medidas conscientes em seus processos, durante todo o ciclo de vida de seus produtos (SUBRAMONIAM; HUISINGH; CHINNAM, 2010). Nesta perspectiva, a logística reversa é uma temática bastante discutida quando se trata de sustentabilidade.

O termo Logística Reversa começou a ser incialmente estudado na década de 70 e 80 , sendo uma de suas definições mais antigas dada por Lambert e Stock (1982). De acordo com o Council of Supply Chain Management Professionals, logística reversa é uma área especializada da logística que foca no movimento de produtos após a venda, incluindo os produtos que são devolvidos, em outras palavras, há uma preocupação com todo o ciclo de vida dos produtos. Entretanto, uma das definições de logística reversa que é mais aceita é a dada por Rogers e Tibben-Lembke (1999) que diz que esta é o processo de planejamento, implementação e controle do fluxo de matérias primas, materiais em processo e informações, desde a origem da cadeia de suprimentos até sua destinação final.

Este estudo assume que decisões organizacionais são influenciadas interna e externamente pelo ambiente em que empresas estão inseridas (YE et al., 2013). Desta forma, é baseado na teoria institucional, para ajudar a compreender como pressões institucionais influenciam no desenvolvimento de práticas sustentáveis com foco na logística reversa.

Devido a uma, ainda existente, falta de entendimento sobre o conceito de institucionalismo e suas aplicações, há um retardo no avanço de uma sociedade que possua uma ampla consciência ambiental, pois ainda existe uma lacuna ao se pensar em como os vários agentes envolvidos, sejam eles, Estado ou empresas, podem motivar seus cidadãos a terem iniciativas ambientais que sejam independentes da regulação já exposta de forma coercitiva (LAM et al., 2019).

Diante da também falta de estudos empíricos que relacionam a Logística Reversa com a teoria institucional, falta esta vista mais fortemente no cenário nacional do que no internacional, pois já se verificam este tipo de estudo em alguns países do mundo, este artigo possui como objetivo, fazer o mapeamento de todos os aspectos e facetas da Logística Reversa sob o olhar da Teoria Institucional, usando uma revisão estruturada da literatura para identificar orientações importantes para pesquisas futuras nesta temática (LEE; VAN WYK, 2015).

A pesquisa se delimita em desenvolver uma análise bibliométrica, ao levantar e analisar pesquisas em inglês de artigos científicos sobre Logística Reversa com o enfoque da Teoria Institucional de revistas da base de dados da Web of Science e Scopus, sem definição de período. Trata-se assim de um problema de pesquisa relevante, pois pesquisas que envolvem a Teoria Institucional ao se analisar a implementação de aspectos sustentáveis na cultura de uma empresa, encontram-se em estágio embrionário, principalmente no Brasil, tendo os Estados Unidos como principal produtor de artigos nesta área. Além disso, a Logística Reversa vem ganhando notoriedade ao tratar de práticas sustentáveis, sendo necessário o estudo deste tema em diferentes perspectivas e enfoques (CHILESHE et al., 2018)

Este trabalho está estruturado em cinco seções, sendo a seção 2 uma fundamentação teórica sobre a Logística Reversa e sua relação com pressões institucionais, a seção 3 um tratamento de questões 
metodológicas da pesquisa, a seção 4 uma exposição dos resultados obtidos com a análise bibliométrica e uma discussão destes e a seção 5 uma apresentação das principais conclusões, sugestões para trabalhos futuros, seguidas das referências bibliográficas que foram utilizadas.

\section{Teoria Institucional}

A aplicação da Teoria Institucional na gestão da cadeia de suprimentos é ainda um campo de estudo não estruturado (LEE; VAN WYK, 2015). Entretanto, de acordo com Kauppi (2013), estudos envolvendo a teoria institucional na gestão da cadeia de suprimentos obtiveram um relativo crescimento nos últimos anos. Tratando-se da teoria institucional e suas origens, a concepção tradicional, racional, baseada em teoria organizacionais anteriores que teve como alguns de seus expoentes autores como Taylor e Fayol, começou a ser desafiada com esta teoria que destaca a relação da organização com o ambiente e como ocorrem estas interações (FAMIYEH; KWARTENG, 2018). Philip Selznick é considerado o precursor da teoria institucional com sua obra Foundations of the theory of Organization de 1948 (SUDDABY, 2010). O autor conceitua a teoria institucional nesta obra que tem como principal conceito a influência que comportamentos sociais, normas e valores, inseridos no ambiente de determinada organização, produzem nas escolhas, decisões e comportamentos destas (FAMIYEH; KWARTENG, 2018). Em outras palavras devido as pressões sociais exercidas pelo ambiente, muitas normas, regras e práticas são aderidas pela organização, o que podem torná-las semelhantes a outras organizações que são expostas ao mesmo conjunto de pressões.

Uma das definições mais famosas da teoria institucional afirma que:

" $\mathrm{Na}$ teoria institucional, as organizações são influenciadas por pressões normativas, ora oriundas de fontes externas, como o Estado, ora oriundas de dentro da própria organização. Sob certas condições, essas pressões levam a organização a ser guiada por elementos legítimos, desde procedimentos operacionais padrão até certificação profissional e exigência do Estado (ZUCKER, 1987, p.443)."

Segundo Meyer e Rowan (1977), essas regras oriundas do ambiente externo funcionam como mitos que propiciam à organização maior legitimidade, estabilidade e chance de sobrevivência dentro do ambiente competitivo, tendo os processos sociais status de norma no pensamento e ações dos indivíduos na sociedade. Tratando-se dos principais trabalhos e expoentes internacionais, que estabeleceram a base do institucionalismo moderno pode-se citar Meyer e Rowan (1977), Dimaggio e Powell (1983), Tolbert e Zucker (1983), Meyer e Scott (1983) (MOTKE; RAVANELLO; RODRIGUES, 2016).

Segundo Carvalho e Vieira (2003), foi na década de 80 que começaram pesquisas com o intuito de clarificar conceitos do processo de institucionalização, no campo do estudo organizacional brasileiro. Entretanto, foi a partir do ano de 1999 que a produção nacional começou a se destacar, ao passo que a produção internacional já estava bastante aquecida, sendo esta usada como base para os estudos brasileiros. Desta maneira, somente a partir de 2001, o campo da teoria institucional nacional mostrou um maior amadurecimento e mais independência em relação a este consumo inicial, adquirindo uma certa autonomia, não deixando de ter uma referência internacional, mas produzindo também, conhecimento local gerado no ambiente em que as organizações estão inseridas. (GUARIDO FILHO, 2008).

A teoria institucional tem sido utilizada em conjunto com muitas outras áreas por diversos autores ao redor do mundo. Na perspectiva sustentável, em que é avaliada como as pressões institucionais influenciam na implementação de aspectos da sustentabilidade, assunto hoje tão debatido, tem-se alguns trabalhos como os de Chu et al. (2017), Khor et al. (2016), Lam et al. (2019) e de Lee e Van Wyk (2015). Deste modo, nota-se que a teoria institucional tem sido utilizada em conjunto com a análise de aspectos sustentáveis e além desta área, na política, na educação e na medição de performance das organizações (DA et al., 2012).

Um conceito importante na teoria institucional é o isomorfismo que se caracteriza por ser um processo 
pelo qual uma organização se assemelha a outras inseridas em um mesmo conjunto de condições ambientais. Desta maneira, são excluídas as formas que são consideradas não ótimas e os gestores passam a tomar decisões que caracterizam a homogeneidade de uma população (DIMAGGIO; POWELL, 2005). Este processo de isomorfismo institucional, de acordo com DiMaggio e Powell (1983), ocorre por meio de três mecanismos principais: pressões coercitivas, normativas e miméticas, estas discutidas a seguir.

\section{Pressões coercitivas}

Pressões coercitivas são aquelas que as organizações sofrem de outras das quais elas são dependentes, podendo ser formais ou informais. Esta dependência pode estar relacionada a recursos materiais, humano ou econômico, entre outros (DIMAGGIO; POWELL, 2005). Em outras palavras, a organização se vê obrigada a adotar alguma regra, prática ou comportamento normalmente expostos pelo Estado ou por organizações que exercem grande poder no ambiente externo (MOTKE; RAVANELLO; RODRIGUES, 2016). Em relação aos aspectos sustentáveis, exemplos de como essas pressões são exercidas podem ser vistas no trabalho de Famiyeh e Kwarteng (2018), que cita alguns órgãos ambientais que regularizam aspectos ambientais em Gana, como a Agência de Proteção Animal, o ministério responsável pelo meio ambiente e as comissões ambientais.

No Brasil, o marco na gestão de resíduos sólidos foi promulgado em 2010, com a Política Nacional dos Resíduos Sólidos (Lei $n^{\circ}$ 12.305) em que são expostos regras e diretrizes para a gestão dos produtos desde sua origem até seu destino. Entre os órgãos ambientais reguladores no Brasil, podese citar o CONAMA e o IBAMA, por exemplo. Na Europa, muitos países possuem regulamentos rígidos em relação a reciclagem e a gestão dos resíduos, contribuindo para a logística reversa.

Desta maneira, regulamentos, leis e órgãos reguladores são exemplos de instrumentos que são usados para exercer a pressão coercitiva nas organizações, obrigando todos os agentes envolvidos na cadeia de suprimentos a implementar a logística reversa, de maneira a influenciar atitudes da alta gerência (YE et al., 2013).

\section{Pressões miméticas}

Pressões miméticas ocorrem quando uma determinada organização toma como base outras organizações para a realização de suas ações, sendo as imitadas bem-sucedidas no seu ramo de atuação (HUANG; YANG, 2014). Tratando-se do aspecto sustentável, organizações tendem a imitar outras que tem a implementação da logística reversa em estágio já avançado e bem-sucedido. De acordo com Dimaggio e Powell (1983), esta pressão nada mais é do que a resposta da organização para incertezas proporcionadas pelo meio ambiente. Um exemplo deste tipo de pressão ocorre quando a organização é questionada sobre alguma característica ou problema que surgiu na implementação da logística reversa e não tem experiência anterior na área, tais empresas tendem a utilizar soluções dadas por outras que foram bem-sucedidas para se legitimar.

Segundo Famiyeh e Kwarteng (2018), as pressões coercitivas e miméticas são as que mais influenciam as organizações. No seu estudo, os autores analisaram empresas em Gana que implementaram práticas sustentáveis devido a regulações ou ao benchmarking que realizaram, para avaliar outras empresas que implementaram práticas sustentáveis de sucesso em seus processos.

\section{Pressões normativas}

Pressões normativas são, de acordo com definição dada por Dimaggio e Powell (1983), ligadas a profissionalização. Os autores destacam que universidades e treinamentos profissionais são importantes no desenvolvimento de normas da organização e na capacitação de gestores e sua equipe. Desta maneira, as pressões normativas se relacionam com os esforços feitos por indivíduos que 
ocupam cargos semelhantes para caracterizar seus processos de trabalhos. Não é à toa que ao surgir determinada vaga de emprego, o perfil do profissional já é traçado e este vem de outra organização em que os processos de trabalho se assemelham a sua antiga função. No caso das práticas sustentáveis, de acordo com Huang e Yang (2014), para a implementação da logística reversa, a principal pressão normativa é derivada do cliente, ou seja, do que estes consideram importantes.

\section{Logística Reversa e sua relação com a Teoria Institucional}

Muitas são as definições dada a Logística Reversa, mas uma das definições mais famosa é formulada por Rogers e Tibben-Lembke (2001) que a define como o processo responsável por planejar, implementar e controlar o fluxo de todos os produtos, serviços e informações que estejam envolvidos nos processos desde o início até o consumo final, em que seja realizado corretamente o descarte do que foi utilizado para que haja agregação de valor no produto final.

De acordo com Lee e Van Wyk (2015), o volume de trabalhos que tratam da logística reversa cresceu vertiginosamente na última década e vários autores se destacam nesta área. Srivastava (2007), tem grande destaque no meio acadêmico, ao tratar especificadamente da gestão verde da Cadeia de Suprimentos. Lu, Wu e Kuo (2007) teve como objetivo principal do seu estudo atender aos critérios de desempenho ambiental entre todos os envolvidos da cadeia de fornecimento, promovendo o comportamento ambiental responsável. Estudos que procuram desenvolver modelos que otimizem a rede logística e que minimizem os custos do fluxo reverso, também estão sendo amplamente estudados por Kim et al. (2007), Pokharel e Mutha (2009) e Govindan et al. (2008).

Apesar dos crescentes trabalhos na área de logística reversa, ainda são poucos os que a trata na perspectiva da Teoria Institucional. Li (2014), relaciona as práticas inovadoras da logística reversa com sua performance. Para este fim, o autor relaciona regulamentos do Governo com pressões coercitivas, demanda do mercado com pressões normativas e competição com pressões miméticas. Ye et al. (2013) estabelecem como as pressões institucionais influenciam na postura dos gestores em relação a implementação da logística reversa.

Neste mesmo ponto de vista, Huang e Yang (2014), relaciona como a logística reversa pode influenciar no desempenho econômico, verificando como as pressões institucionais moderam este relacionamento. Lee e Van Wyk (2015) mostraram que seis constructos institucionais, risco político, risco financeiro, liberdade econômica, liberdade política e competitividade, influenciam direta ou indiretamente o desempenho logístico. Huang, Yang e Wong (2016), analisam como as pressões institucionais afetam o gerenciamento e prática de retornos, além da recuperação de produtos. Seguindo as mesmas ideias, alguns autores exploraram como fatores internos e externos levam as empresas a adotar a cadeia de suprimentos verde, destacando pressões coercitivas, miméticas e normativas. (CHU et al., 2017; DUBEY et al., 2017; FAMIYEH; KWARTENG, 2018; HUANG; HUANG; YANG, 2017; KAUPPI, 2013; KHOR et al., 2016; LAM et al., 2019; LI et al., 2019).

Duas bases de pesquisa foram utilizadas no estudo, Web of Science e Scopus. No geral, vinte e dois artigos foram encontrados ao se pesquisar na Web of Science, com as palavras chaves Institutional Theory and Reverse Logistics. Já na base Scopus, com os mesmos critérios de pesquisa, foram encontrados somente dois artigos que não estavam duplicados na Web of Science. O Quadro 1, sintetiza os 24 artigos com seus respectivos autores, revistas e como é aplicada a teoria institucional nestes. 
Quadro 1 - Artigos que aplicam a teoria institucional na logística reversa

\begin{tabular}{|c|c|c|}
\hline Autor & Revista & Uso da Teoria Institucional \\
\hline Chu et al. (2017) & Sustainability & $\begin{array}{l}\text { Investigação de como pressões do } \\
\text { governo, clientes e concorrentes } \\
\text { influenciam no desempenho ambiental } \\
\text { e operacional das empresas. }\end{array}$ \\
\hline Dubey et al. (2017) & $\begin{array}{l}\text { International Journal of } \\
\text { Production Economics }\end{array}$ & $\begin{array}{l}\text { Investigação de como as pressões } \\
\text { institucionais influenciam a } \\
\text { performance das organizações e como } \\
\text { o efeito destas moderam a cultura } \\
\text { organizacional. }\end{array}$ \\
\hline
\end{tabular}

International Journal of

Productivity and

Performance

Management

Famiyeh e Kwarteng (2018)

Industrial Marketing

Management

Geng et al. (2017)

Gollnhofer e Turkina (2015)

Cross Cultural

Management

International Journal of

Operations and

Production

Management

Hsu et al. (2013)

Huang e Yang (2014)

Management Research Review
Apontamento das pressões coercitivas e miméticas como as principais forças que influenciam empresas em Gana.

Desenvolvimento de uma estrutura que caracteriza os direcionadores e barreiras ao implementar a logística reversa em países asiáticos.

De acordo com o estudo, as dimensões regulatória, cognitiva e normativa têm um efeito direto na escolha do modo de entrada no mercado.

Análise dos fatores que motivam as empresas a adotar o gerenciamento da cadeia de suprimentos verde.

Identificação de como pressões institucionais moderam a relação entre inovação em logística reversa e performance econômica.

International Journal of Exploração de como fatores internos e Physical Distribution externos levam as empresas a adotar

Huang, Huang e Yang (2017) and Logistics Management ações de logística reversa baseando-se na teoria institucional.

\begin{tabular}{lll}
\hline Huang, Yang e Wong (2016) & $\begin{array}{l}\text { Supply } \\
\text { Management }\end{array}$ & Chain \\
\hline
\end{tabular}

Exploração das relações entre pressões institucionais, comprometimento de recursos e gestão de retornos.

Investigação do papel moderador da

Khor et al. (2016)

International Journal of Production Economics

Resources,

Conservation Recycling

Industrial Management and Data Systems

Service Business pressão regulatória na relação entre a logística reversa e os níveis de desempenho.

Validação de como as pressões and coercitivas, miméticas e normativas influenciam a vida dos indivíduos.

Análise da implantação de práticas sustentáveis e como estas influenciam o desempenho econômico e ambiental.

Demonstração de que risco político, risco financeiro, liberdade econômica, liberdade política e competitividade 


\begin{tabular}{|c|c|c|}
\hline & & $\begin{array}{l}\text { têm influências no desempenho } \\
\text { logístico }\end{array}$ \\
\hline Li (2014) & $\begin{array}{l}\text { Journal of Cleaner } \\
\text { Production }\end{array}$ & $\begin{array}{l}\text { O estudo procura examinar as ligações } \\
\text { entre pressões institucionais, práticas } \\
\text { de inovação ambiental e desempenho. }\end{array}$ \\
\hline Li et al. (2019) & $\begin{array}{l}\text { International Journal of } \\
\text { Operations and } \\
\text { Production } \\
\text { Management }\end{array}$ & $\begin{array}{l}\text { Investigação de como pressões } \\
\text { externas e internas influenciam o } \\
\text { comportamento da alta gerência na } \\
\text { aplicação de práticas sustentáveis. }\end{array}$ \\
\hline Liu, Zhang e Ye (2018) & $\begin{array}{l}\text { Journal } \\
\text { Environmental } \\
\text { Management }\end{array}$ & $\begin{array}{l}\text { Exploração de como as relações de } \\
\text { poder dos fornecedores influenciam a } \\
\text { adoção de práticas de sustentabilidade. }\end{array}$ \\
\hline Malviya, Kant e Gupta (2018) & $\begin{array}{l}\text { Business Strategy and } \\
\text { the Environment }\end{array}$ & $\begin{array}{l}\text { Estudo de como pressões institucionais } \\
\text { ajudam os gerentes a tomar decisões. }\end{array}$ \\
\hline Sarkis, Gonzalez-Torre e Adenso-Diaz (2010) & $\begin{array}{l}\text { Journal of Operations } \\
\text { Management }\end{array}$ & $\begin{array}{l}\text { Exploração de como as pressões de } \\
\text { partes interessadas influenciam na } \\
\text { adoção de práticas ambientais. }\end{array}$ \\
\hline Shaharudin et al. (2015) & $\begin{array}{l}\text { Journal of Cleaner } \\
\text { Production }\end{array}$ & $\begin{array}{l}\text { Desenvolvimento de propostas para o } \\
\text { gerenciamento de devoluções de } \\
\text { produtos usando a Teoria Institucional. }\end{array}$ \\
\hline Shaharudin, Zailani e Ismail (2015) & $\begin{array}{l}\text { International Journal of } \\
\text { Management Practice }\end{array}$ & $\begin{array}{l}\text { A teoria Institucional foi usada para } \\
\text { fornecer uma base de explicação para o } \\
\text { uso de terceiros na logística reversa. }\end{array}$ \\
\hline Syazwan et al. (2016) & $\begin{array}{l}\text { Journal of Islamic } \\
\text { Marketing }\end{array}$ & $\begin{array}{l}\text { Demonstração que o apoio do governo } \\
\text { pode ser um moderador da relação } \\
\text { entre a logística de Halal e o seu } \\
\text { desempenho. }\end{array}$ \\
\hline Vlachos (2016) & $\begin{array}{l}\text { International Journal of } \\
\text { Logistics Research and } \\
\text { Applications }\end{array}$ & $\begin{array}{l}\text { Demonstração de como alguns fatores } \\
\text { institucionais são mais significativos do } \\
\text { que fatores da cadeia de suprimentos. }\end{array}$ \\
\hline Wan, Yuan e Lai (2019) & $\begin{array}{l}\text { Journal of Enterprise } \\
\text { Information } \\
\text { Management }\end{array}$ & $\begin{array}{l}\text { Exploração de como pressões externas } \\
\text { influenciam na terceirização logística. }\end{array}$ \\
\hline Ye et al. (2013) & $\begin{array}{l}\text { International Journal of } \\
\text { Production Economics }\end{array}$ & $\begin{array}{l}\text { Estudo de como pressões institucionais } \\
\text { influenciam na postura dos gerentes em } \\
\text { relação à implementação da logística } \\
\text { reversa. }\end{array}$ \\
\hline
\end{tabular}

Fonte: Autor.

\section{Procedimentos metodológicos}

A pesquisa se caracteriza por ser de caráter exploratório que utiliza recursos bibliométricos ao se levantar e analisar pesquisas em inglês de artigos científicos sobre Logística Reversa com o enfoque da Teoria Institucional de revistas da base de dados da Web of Science e Scopus, com as palavras chaves Institutional Theory and Reverse Logistics, sem restrição do tempo. De acordo com Pritchard (1969), o estudo bibliométrico tem como objetivo, estudar o estado da ciência, em que um conjunto de leis e princípios são utilizadas para tal tarefa, oferecendo aos dados um tratamento quantitativo.

Todos os 24 artigos encontrados em ambos as bases tiveram seus resumos lidos, para verificar se estes tratavam de assuntos relacionados a Logística Reversa utilizando a teoria institucional.

Apresenta-se na Figura 1, as etapas associadas ao desenvolvimento desta pesquisa. 


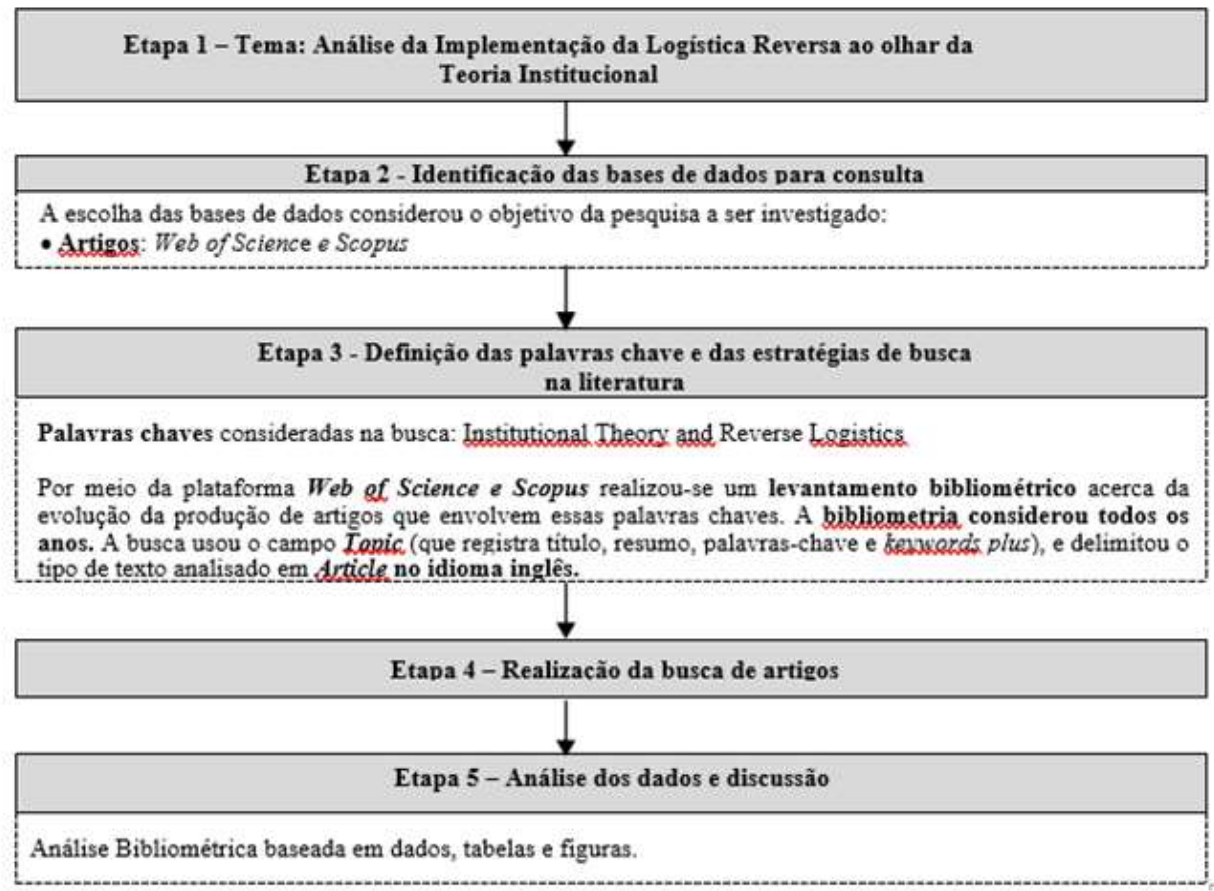

Figura 1 - Visão Esquemática do Método Fonte: Autor.

Desta maneira, percebe-se que a metodologia da análise bibliométrica foi dividida em cinco passos principais.

\section{Apresentação e análise dos resultados}

Foram utilizadas duas bases de dados na análise bibliométrica, a Web of Science e o Scopus, sendo que na Web of Science foram encontrados 22 artigos, no idioma inglês. Já na Scopus, foram encontrados 7, porém 5 deles já estavam incluídos na busca anterior na Web of Science. Desta maneira, abordará a análise e discussão dos 24 artigos identificados, com as palavras chaves Institutional Theory and Reverse Logistics, sem restrição de tempo.

No intuito de atingir o objetivo do artigo, este item será subdividido em três seções, a primeira parte será destinada a apresentar informações gerais dos artigos para contextualizar a análise, a segunda apresenta os periódicos em destaque e a terceira o mapeamento geral dos autores. Abordará então, a análise e discussão dos 24 artigos identificados.

\section{Informações gerais}

Esta seção é caracterizada por contextualizar os artigos pesquisados, expondo a evolução temporal da logística reversa na perspectiva da teoria institucional, os países que mais publicam e qual a abordagem e métodos utilizados nas pesquisas.

\section{Evolução Temporal}

Para uma breve contextualização do tema, no sentido de demonstrar os anos de publicação, para poder visualmente identificar quais os anos de mais produção acadêmica e se estas estão crescendo ao longo do tempo, foi elaborada a Figura 2, que caracteriza o número de artigos ao longo dos anos nas bases de dados da Web of Science e Scopus. 


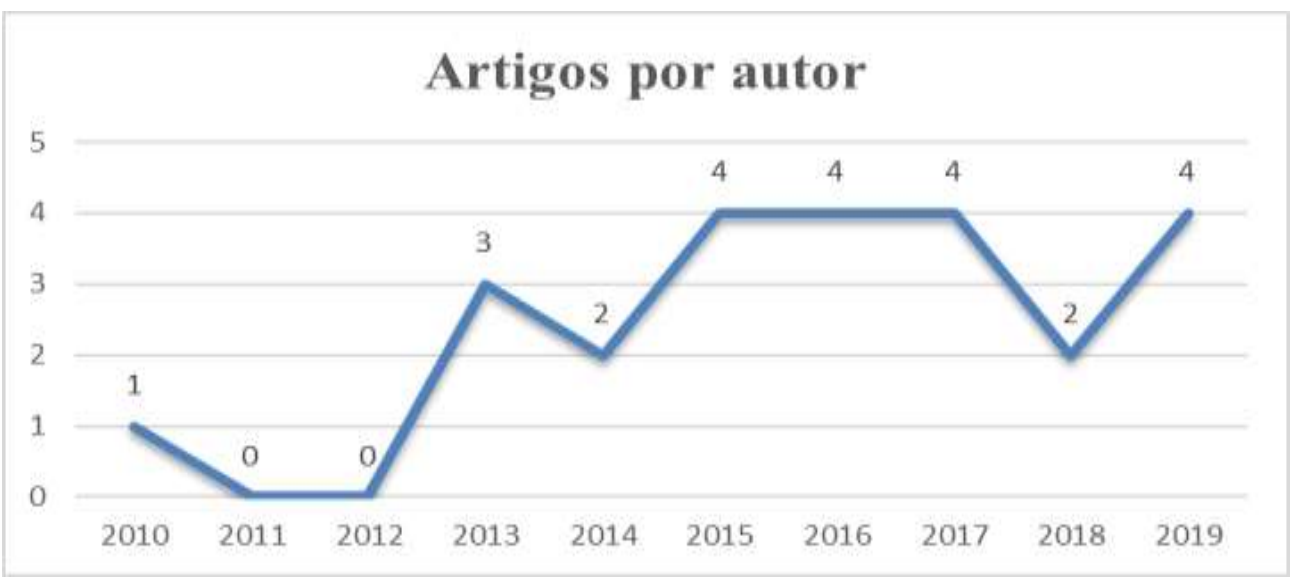

Figura 2 - Evolução Temporal do número de artigos

Fonte: Elaboração própria, a partir dos dados coletados.

Pode-se perceber que as publicações, com os critérios que foram definidos, começaram no ano de 2010, seguidos de uma recessão em 2011 e 2012, um pico em 2013 e uma pequena queda em 2014. Em 2015, 2016 e 2017 a produção se manteve estável em sua maior quantidade de artigos até o momento, seguido de uma redução pela sua metade em 2018. Em 2019, a quantidade de artigos volta a ser quatro, mostrando uma tendência de crescimento nos próximos anos.

\section{Origem das pesquisas}

No total, ao se analisar os artigos pesquisados obteve-se 43 pesquisadores, sejam eles, autores ou coautores. Destes 43 autores, 28\% são de instituições americanas, o que mostra que em sua maioria, estudos acadêmicos que tem como foco a logística reversa aliada a teoria institucional encontram-se nos Estados Unidos, fato demonstrado na Tabela 1.

Tabela 1 - Origem das pesquisas

\begin{tabular}{lll}
\hline País de origem & Número de autores & Porcentagem \\
\hline Estados unidos & 12 & $28 \%$ \\
\hline China & 7 & $16 \%$ \\
\hline Inglaterra & 6 & $14 \%$ \\
\hline Malásia & 4 & $9 \%$ \\
\hline Taiwan & 4 & $9 \%$ \\
\hline Canadá & 1 & $2 \%$ \\
\hline Dinamarca & 1 & $2 \%$ \\
\hline França & 1 & $2 \%$ \\
\hline Gana & 1 & $2 \%$ \\
\hline Índia & 1 & $2 \%$ \\
\hline Coréia do sul & 1 & $2 \%$ \\
\hline Espanha & 1 & $2 \%$ \\
\hline Suíça & 1 & $2 \%$ \\
\hline Tailândia & 2 & $5 \%$ \\
\hline
\end{tabular}

Fonte: Elaboração própria, a partir dos dados coletados.

Com uma breve análise da Tabela 1, percebe-se que os três principais países que mais publicam seriam 
os Estados Unidos, China e Inglaterra, respectivamente. Porém, observa-se que a teoria institucional vem sendo pesquisada com afinco na Malásia e no Taiwan. Estudos brasileiros não foram identificados com os critérios de pesquisa utilizados.

\section{Abordagem das pesquisas}

O próximo aspecto analisado foi a abordagem das pesquisas, se esta é feita de maneira quantitativa, qualitativa ou de maneira mista. Esta análise pode ser vista na Tabela 2.

Tabela 2 - Abordagem das pesquisas

\begin{tabular}{lcc}
\hline Abordagem da pesquisa & Artigos & Porcentagem \\
\hline Qualitativa & 5 & $21 \%$ \\
\hline Quantitativa & 17 & $71 \%$ \\
\hline Quanti-quali & 2 & $8 \%$ \\
\hline \multicolumn{2}{c}{ Fonte: Elaboração própria, a partir dos dados coletados. }
\end{tabular}

Analisando a Tabela 2, pode-se constatar que $71 \%$ dos artigos utilizam a abordagem quantitativa, seguidos de $21 \%$ que utilizam a abordagem qualitativa e $8 \%$ que utilizam a abordagem mista, ou seja, a abordagem quanti-quali.

\section{Métodos das pesquisas utilizados}

Os métodos de pesquisa encontrados nos artigos foram revisão da literatura, documental e entrevista, survey e estudo de caso com uso de múltiplas técnicas. Ao se fazer a análise dos métodos de pesquisa, procura-se identificar os procedimentos metodológicos que são mais frequentes ao abordar a temática da logística reversa sob o olhar da teoria institucional. A quantidade de artigos que usam cada método encontra-se na Tabela 3.

Tabela 3 - Métodos das pesquisas

\begin{tabular}{lcc}
\hline Método de pesquisa & Artigos & Porcentagem \\
\hline Revisão da Literatura & 2 & $8 \%$ \\
\hline Documental e Entrevista & 1 & $4 \%$ \\
\hline Survey & 16 & $67 \%$ \\
\hline Estudo de Caso: múltiplas técnicas & 5 & $21 \%$ \\
\hline
\end{tabular}

Pela Tabela 3, tem-se o survey como o método de pesquisa mais utilizado nos artigos analisados.

\section{Periódicos de destaque}

Ao se analisar a Tabela 4, foram identificados 24 periódicos que publicaram artigos envolvendo a logística reversa e a teoria institucional. 
Logística reversa e Teoria Institucional: um estudo bibliométrico

Tabela 4 - Periódicos em destaque

\begin{tabular}{|c|c|c|}
\hline Periódico & Artigos & Porcentagem \\
\hline $\begin{array}{l}\text { INTERNATIONAL JOURNAL OF PRODUCTION } \\
\text { ECONOMICS }\end{array}$ & 3 & $13 \%$ \\
\hline $\begin{array}{l}\text { INTERNATIONAL JOURNAL OF OPERATIONS } \\
\text { PRODUCTION MANAGEMENT }\end{array}$ & 2 & $8 \%$ \\
\hline JOURNAL OF CLEANER PRODUCTION & 2 & $8 \%$ \\
\hline BUSINESS STRATEGY AND THE ENVIRONMENT & 1 & $4 \%$ \\
\hline $\begin{array}{l}\text { CROSS CULTURAL MANAGEMENT AN INTERNATIONAL } \\
\text { JOURNAL }\end{array}$ & 1 & $4 \%$ \\
\hline INDUSTRIAL MANAGEMENT DATA SYSTEMS & 1 & $4 \%$ \\
\hline INDUSTRIAL MARKETING MANAGEMENT & 1 & $4 \%$ \\
\hline $\begin{array}{l}\text { INTERNATIONAL JOURNAL OF LOGISTICS RESEARCH } \\
\text { AND APPLICATIONS }\end{array}$ & 1 & $4 \%$ \\
\hline 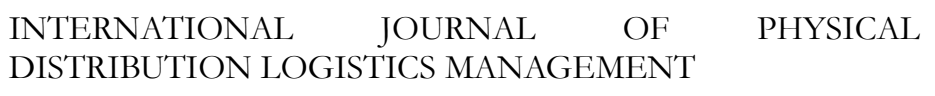 & 1 & $4 \%$ \\
\hline $\begin{array}{l}\text { INTERNATIONAL JOURNAL OF PRODUCTIVITY AND } \\
\text { PERFORMANCE MANAGEMENT }\end{array}$ & 1 & $4 \%$ \\
\hline $\begin{array}{l}\text { JOURNAL OF } \quad \text { ENTERPRISE } \\
\text { MANAGEMENT }\end{array}$ & 1 & $4 \%$ \\
\hline JOURNAL OF ENVIRONMENTAL MANAGEMENT & 1 & $4 \%$ \\
\hline JOURNAL OF ISLAMIC MARKETING & 1 & $4 \%$ \\
\hline JOURNAL OF OPERATIONS MANAGEMENT & 1 & $4 \%$ \\
\hline RESOURCES CONSERVATION AND RECYCLING & 1 & $4 \%$ \\
\hline SERVICE BUSINESS & 1 & $4 \%$ \\
\hline $\begin{array}{l}\text { SUPPLY CHAIN MANAGEMENT AN INTERNATIONAL } \\
\text { JOURNAL }\end{array}$ & 1 & $4 \%$ \\
\hline SUSTAINABILITY & 1 & $4 \%$ \\
\hline $\begin{array}{l}\text { INTERNATIONAL JOURNAL OF MANAGEMENT } \\
\text { PRACTICE }\end{array}$ & 1 & $4 \%$ \\
\hline MANAGEMENT RESEARCH REVIEW & 1 & $4 \%$ \\
\hline
\end{tabular}

Fonte: Elaboração própria, a partir dos dados coletados.

Os periódicos que mais publicaram artigos nesta temática foram o International Journal of Production Economics, o International Journal of Operations Production Management e o Journal of Cleaner Production com $13 \%, 8 \%$ e $8 \%$ de todos os artigos analisados, respectivamente.

\section{Perfil dos autores}

Esta seção será responsável por mapear o perfil dos autores, destacando os que mais publicam, suas áreas de pesquisa e a instituição e vínculo destes. Inicialmente foi demonstrado na Figura 3, os dez autores que mais publicaram na área de logística reversa relacionada a teoria institucional. 


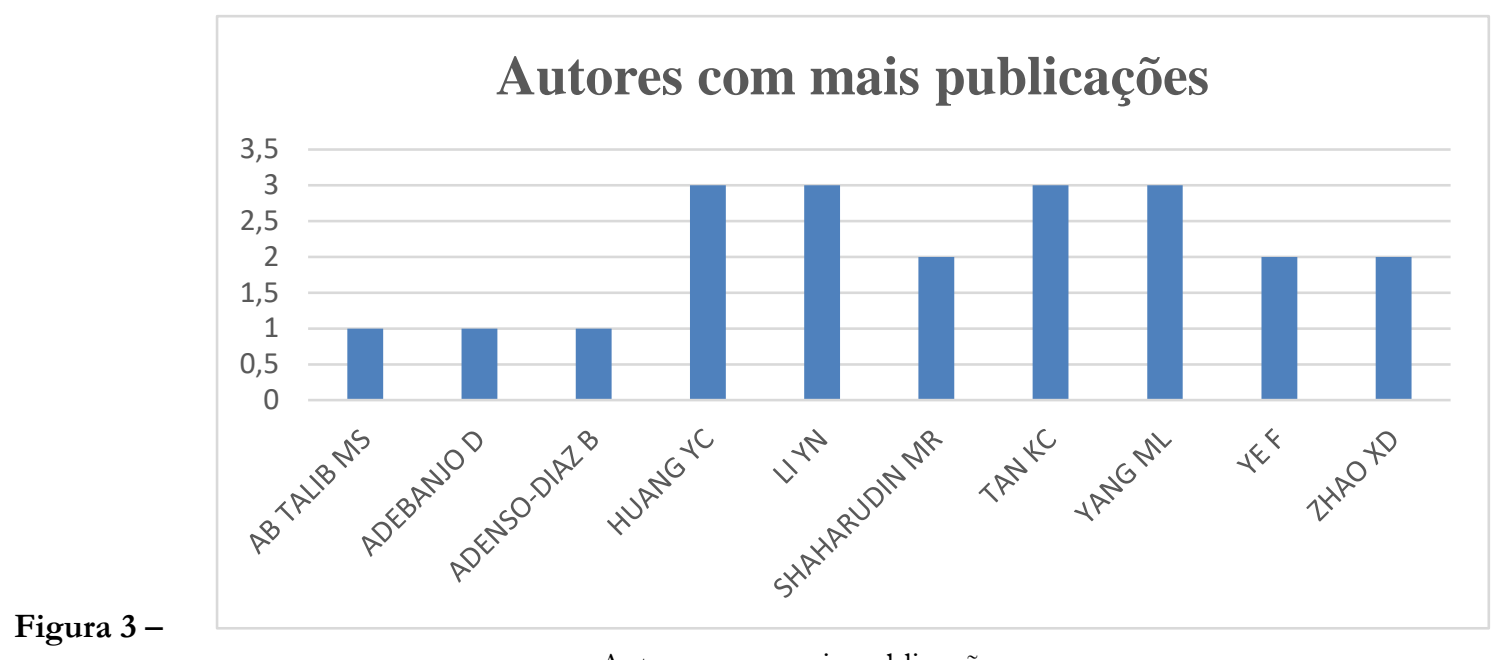

\section{Autores com mais publicações}

Autores com mais publicações

Fonte: Elaboração própria, a partir dos dados coletados.

Pela análise da Figura 3, observa-se quatro autores com três artigos cada e três autores com dois artigos, seguidos de três autores com um artigo.

\section{Áreas com mais artigos publicados}

A Figura 4 retrata as principais áreas de estudo dos autores, sendo que 15 artigos são referentes a área de negócios.

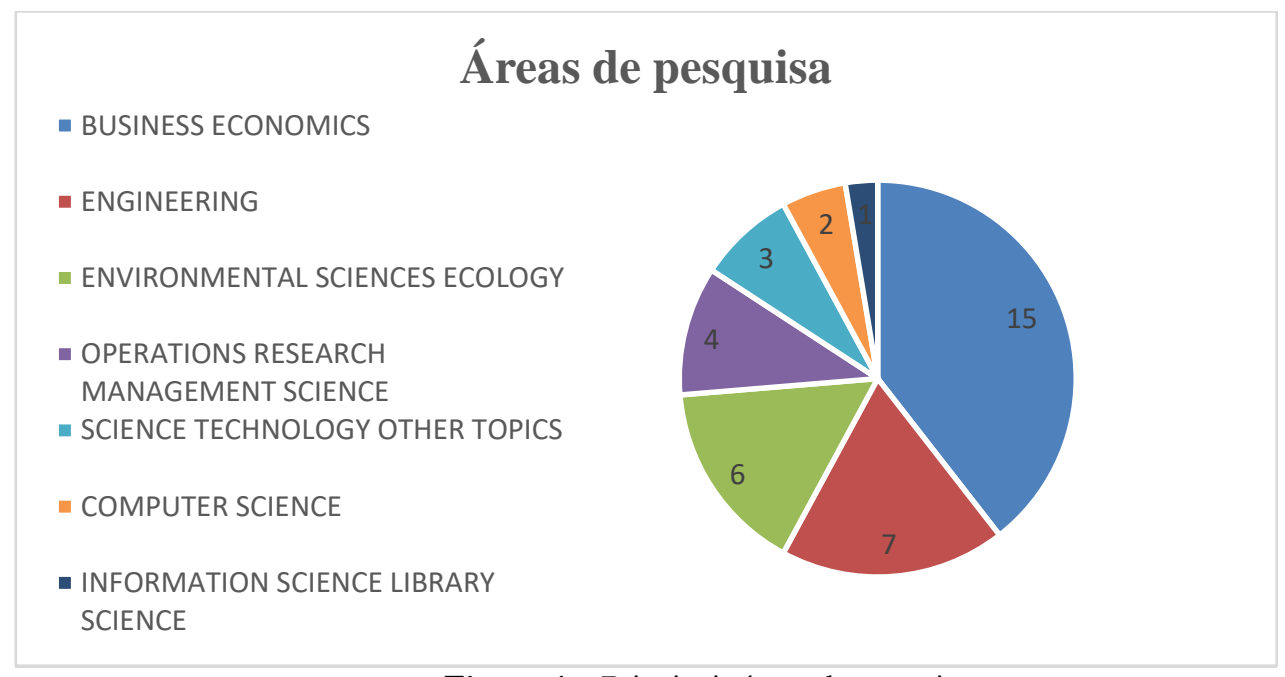

Figura 4 - Principais áreas de pesquisa

Fonte: Elaboração própria, a partir dos dados coletados.

No total foram estabelecidas sete áreas principais para a análise dos 24 artigos encontrados.

\section{Instituições e Vínculos}

Em relação às instituições e aos respectivos vínculos dos autores que contribuíram com a discussão em nível internacional, pela ausência de trabalhos nacionais com os critérios pesquisados, podem-se destacar 24, que juntas somam 52\% dos autores, conforme Figura 5 e Tabela 5. 


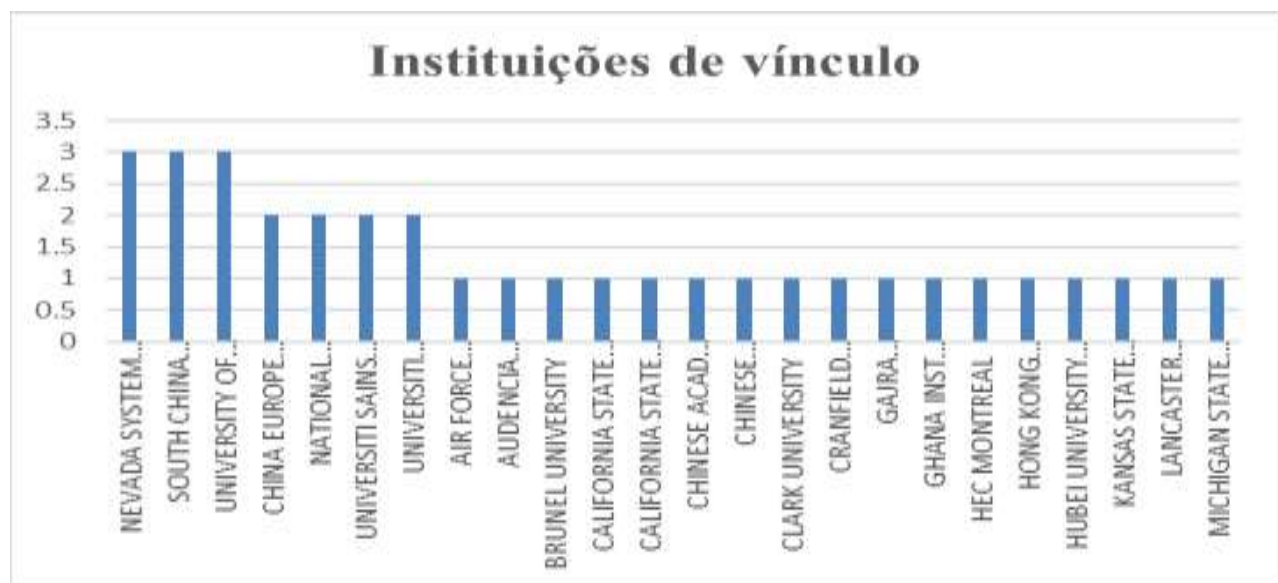

Figura 5 - Instituições dos autores

Fonte: Elaboração própria, a partir dos dados coletados.

Tabela 5 - Principais Instituições dos autores

\begin{tabular}{|c|c|c|}
\hline Periódico & Artigos & Porcentagem \\
\hline NEVADA SYSTEM OF HIGHER EDUCATION NSHE & 3 & $5 \%$ \\
\hline SOUTH CHINA UNIVERSITY OF TECHNOLOGY & 3 & $5 \%$ \\
\hline UNIVERSITY OF NEVADA LAS VEGAS & 3 & $5 \%$ \\
\hline CHINA EUROPE INTERNATIONAL BUSINESS SCHOOL & 2 & $3 \%$ \\
\hline $\begin{array}{l}\text { NATIONAL KAOHSIUNG UNIVERSITY OF SCIENCE } \\
\text { TECHNOLOGY }\end{array}$ & 2 & $3 \%$ \\
\hline UNIVERSITI SAINS MALAYSIA & 2 & $3 \%$ \\
\hline UNIVERSITI TEKNOLOGI MALAYSIA & 2 & $3 \%$ \\
\hline AIR FORCE INSTITUTE OF TECHNOLOGY AFIT & 1 & $2 \%$ \\
\hline AUDENCIA BUSINESS SCH & 1 & $2 \%$ \\
\hline BRUNEL UNIVERSITY & 1 & $2 \%$ \\
\hline CALIFORNIA STATE UNIVERSITY BAKERSFIELD & 1 & $2 \%$ \\
\hline CALIFORNIA STATE UNIVERSITY SYSTEM & 1 & $2 \%$ \\
\hline CHINESE ACAD ENVIRONM PLANNING & 1 & $2 \%$ \\
\hline CHINESE UNIVERSITY OF HONG KONG & 1 & $2 \%$ \\
\hline CLARK UNIVERSITY & 1 & $2 \%$ \\
\hline CRANFIELD UNIVERSITY & 1 & $2 \%$ \\
\hline GAJRA DIFFERENCIAL GEARS PVT LTD & 1 & $2 \%$ \\
\hline GHANA INST MANAGEMENT PUBL ADM & 1 & $2 \%$ \\
\hline HEC MONTREAL & 1 & $2 \%$ \\
\hline HONG KONG POLYTECHNIC UNIVERSITY & 1 & $2 \%$ \\
\hline HUBEI UNIVERSITY OF ECONOMICS & 1 & $2 \%$ \\
\hline KANSAS STATE UNIVERSITY & 1 & $2 \%$ \\
\hline LANCASTER UNIVERSITY & 1 & $2 \%$ \\
\hline MICHIGAN STATE UNIVERSITY & 1 & $2 \%$ \\
\hline
\end{tabular}

Fonte: Elaboração própria, a partir dos dados coletados. 
Ao analisar todas estas seções percebe-se um crescimento na temática da logística reversa aliada a teoria institucional devido a uma maior conscientização da população e de todos os agentes envolvidos na vida útil dos produtos, entretanto a produção acadêmica que considera como a teoria institucional pode resultar na implementação da logística reversa ainda não é vista em grande número.

\section{Considerações finais}

O ano de 2019 juntamente com os anos de 2015, 2016 e 2017 foram os de maior produção acadêmica neste tema, tendo os Estados Unidos como principal país produtor na área. A abordagem quantitativa esteve presente em $71 \%$ dos artigos analisados tendo o método de pesquisa do survey em $67 \%$ destes. O International Journal of Production economics produziu 13\% dos artigos, sendo que os autores Yi-Chun Huang, Yina Li, Keah Choon Tan e Min-Li Yang foram responsáveis por 3 artigos cada no período analisado, que apesar de não ter sido especificado, teve início com a produção acadêmica em 2010 se estendendo a 2019. Observa-se também, que a instituição de ensino Nevada System of Higher Education Nshe, South China University of Technology e a University of Nevada Las Vegas totalizaram juntas, 15\% das pesquisas em análise.

Desta maneira, o objetivo do artigo foi atingido, pois foi realizado um mapeamento ao se fazer uma busca de artigos com as palavras chaves Institutional Theory and Reverse Logistics no idioma inglês na base de dados da Web of Science e Scopus. Assim, a revisão estruturada da literatura sob a ótica da Teoria Institucional, identificou que os maiores assuntos desta que são estudados em logística reversa seriam como as pressões coercitivas, miméticas e normativas influenciam ao se implementar a logística reversa nas empresas.

Em relação ao cenário nacional, não foi encontrado nenhum trabalho que analisasse como a teoria institucional exerce influência na logística reversa nas duas bases de dados utilizadas. Como oportunidade de pesquisa, destaca-se a grande variedade do cenário brasileiro em relação a muitas empresas que podem ser analisadas, assuntos como a institucionalização ocorre e como fatores internos e externos influenciam este processo.

Em relação ao cenário internacional, de acordo com a Figura 6, pode-se ver que entre os 24 artigos, somente dois, Shaharudin et al. (2015) e Geng et al. (2017), tiveram como métodos de pesquisa a revisão da literatura. Em sua maioria, os artigos foram abordados de maneira quantitativa, utilizandose de técnicas estatísticas. Assim, este trabalho possui sua relevância ao se diferenciar da maioria dos autores citados ao longo do artigo, que tem como foco mostrar evidências quantitativas para analisar empresas diversas ao redor do mundo. Com isto, colabora ao fortalecer o mapeamento destas duas temáticas, que vem ganhando destaque devido a uma maior conscientização mundial dada a aspectos sustentáveis.

Com a revisão sistemática da literatura, identifica-se importantes orientações para pesquisas futuras nas temáticas da teoria institucional e da logística reversa, o que serve como base para outras pesquisas. Como recomendações futuras, pesquisas podem ser feitas com mais bases de dados, além de estudos de casos, em diferentes setores da economia com diversas organizações.

\section{Referências}

CARVALHO, C. A.; VIEIRA, M. M. F. (Orgs.). Organizações, cultura e desenvolvimento local: a agenda de pesquisa do Observatório da Realidade Organizacional. Recife: Editora Universitária UFPE, 2003. p. 23-40.

CHILESHE, N. et al. Factors driving the implementation of reverse logistics: A quantified model for the construction industry. Waste Management, v. 79, p. 48-57, 2018.

CHU, S. H. et al. The impact of institutional pressures on green supply chain management and firm performance: Top management roles and social capital. Sustainability (Switzerland), v. 9, n. 5, 2017. 
DA, A. T. C. et al. Teoria Institucional. Revista Eletrônica de Gestão Organizacional, v. 10, p. 469496, 2012.

DIMAGGIO, P. J.; POWELL, W. W. GRIMALDI Evolution of the Insects.pdf. American Sociological Review, v. 48, n. 2, p. 147-160, 1983.

DIMAGGIO, P. J.; POWELL, W. W. A gaiola de ferro revisitada: isomorfismo institucional e racionalidade coletiva nos campos organizacionais. RAE - Revista de Administração de Empresas, v. 45, n. 2, p. 74-89, 2005.

DUBEY, R. et al. Examining the effect of external pressures and organizational culture on shaping performance measurement systems (PMS) for sustainability benchmarking: Some empirical findings. International Journal of Production Economics, v. 193, p. 63-76, 2017.

FAMIYEH, S.; KWARTENG, A. Implementation of Environmental Management Practices in Ghanaian Firms. International Journal of Productivity and Performance Management, v. Accepted, 2018.

GOVINDAN, K.; AZEVEDO, G.S.; CARVALHO, H.; MACHADO, C.V. Impact of supply chain management practices on sustainability. Journal of Cleaner Production, v. 85, p. 212-225, 2008.

GUARIDO FILHO, E. R. A construção da teoria institucional nos estudos organizacionais no Brasil: o período 1993-2007. n. May, p. 299, 2008.

HUANG, Y. C.; HUANG, C. H.; YANG, M. L. Drivers of green supply chain initiatives and performance: Evidence from the electrical and electronics industries in Taiwan. International Journal of Physical Distribution and Logistics Management, v. 47, n. 9, p. 796-819, 2017.

HUANG, Y. C.; YANG, M. L. Reverse logistics innovation, institutional pressures and performance. Management Research Review, v. 37, n. 7, p. 615-641, 2014.

HUANG, Y. C.; YANG, M. L.; WONG, Y. J. Institutional pressures, resources commitment, and returns management. Supply Chain Management, v. 21, n. 3, p. 398-416, 2016.

KAUPPI, K. Extending the use of institutional theory in operations and supply chain management research: Review and research suggestions. International Journal of Operations and Production Management, v. 33, n. 10, p. 1318-1345, 2013.

KHOR, K. S. et al. Reverse logistics in Malaysia: The Contingent role of institutional pressure. International Journal of Production Economics, v. 175, p. 96-108, 2016.

KIM, K.; SONG, I.; KIM, J.; JEONG, B. Supply planning model for remanufacturing system in reverse logistics system. Computers \& Industrial Engineering, v. 51, p. 279-287, 2007.

LAM, M. M. L. et al. Effects of institutional environmental forces on participation in environmental initiatives. Resources, Conservation and Recycling, v. 150, n. December 2018, p. 104402, 2019.

LAMBERT, D.M.; STOCK, J.R. Strategic Physical Distribution Management. Irwin, Homewood, IL,1982.

LEE, S. H.; VAN WYK, J. National institutions and logistic performance: a path analysis. Service Business, v. 9, n. 4, p. 733-747, 2015.

LI, Y. Environmental innovation practices and performance: Moderating effect of resource commitment. Journal of Cleaner Production, v. 66, p. 450-458, 2014.

LI, Y. et al. The adoption of green practices by Chinese firms: Assessing the determinants and effects of top management championship. International Journal of Operations and Production Management, 2019. 
LU, Y. Y. L; WU, C. H.; KUO, T. C. Environmental principles applicable to green supplier evaluation by using multi-objective decision analysis. International Journal of Production Research, v. 45, p. 4317-4331, 2007.

MEYER, J. W.; ROWAN, B. Institutionalized Organizations: Formal Structure as Myth and Ceremony. American Journal of Sociology, v. 83, n. 2, p. 340-363, 1977.

MEYER, J. W.; SCOTT, W. R. Organizational Environments: Ritual and Rationality Beverly Hills: Sage, 1983.

MOTKE, F. D.; RAVANELlO, F. DA S.; RODRIGUES, G. O. Teoria Institucional: Um Estudo Bibliométrico Da Última Década Na Web of Science. Contextus - Revista Contemporânea de Economia e Gestão, v. 14, n. 2, p. 63-86, 2016.

POKHAREL, S.; MUTHA, A. Perspectives in reverse logistics: A review. Resources, Conservation and Recycling, v. 53, n. 4, p. 175-182, abr. 2009.

PRITCHARD, A. Statistical bibliography or bibliometrics? Journal of Documentation, v. 25,

n. 4, p. 348-349, 1969.

ROGERS, D. S.; TIBBEN-LEMBKE, R. S. Going Backwards: Reverse Logistics Trends and Practices. [s.l.] Reverse Logistics Executive Council, 1999.

Selznick, P. (1957), Leadership in Administration: A Sociological Perspective, Harper \& Row, New York, NY.

SRIVASTAVA, S. K. Green Supply Chain Management: A State-of-the-Art Literature Review. International Journal of Management Reviews, v. 9, n. 1, p. 53-80, 2007.

SUBRAMONIAM, R.; HUISINGH, D.; CHINNAM, R. B. Aftermarket remanufacturing strategic planning decision-making framework: Theory \& practice. Journal of Cleaner Production, v. 18, n. 16-17, p. 1575-1586, 2010.

SUDDABY, R. Challenges for institutional theory. Journal of Management Inquiry, v. 19, n. 1, p. 14-20, 2010.

TOLBERT, P. S.; ZUCKER, L. G. Institutional Sources of Change in the Formal Structure of Organizations: The Diffusion of Civil Service Reform, 1880-1935. Administrative Science Quarterly, v. 28, n. 1, p. 22, 1983.

YE, F. et al. The impact of institutional pressures, top managers' posture and reverse logistics on performance - Evidence from China. International Journal of Production Economics, v. 143, n. 1, p. 132-143, 2013.

ZUCKER, L. G. Institutional theories of organization. Annual review of sociology. Vol. 13, p. 443464, 1987. 